\title{
SERVICIOS DE ORIENTACIÓN SOCIOLABORAL EN LA REGIÓN DE MURCIA, ESPAÑA: BUENAS PRACTICAS Y DEBILIDADES
}

\section{SOCIOLABOR GUIDANCE SERVICES IN THE REGION OF MURCIA, SPAIN: GOOD PRACTICES AND WEAKNESSES}

\author{
María Fe Sánchez-García* \\ María del Carmen García-García** \\ Magdalena Suárez Ortega ${ }^{* * *}$
}

RESUMEN

Los servicios de orientación sociolaboral constituyen un gran apoyo para la inserción laboral-profesional de las personas demandantes de empleo. Esta investigación se propone indagar acerca de la valoración que merecen los distintos elementos que configuran las buenas prácticas de orientación y las debilidades en los servicios de empleo de la Región de Murcia (España), a partir de las opiniones de personas usuarias y de profesionales de la Orientación. Para ello, se aplica un cuestionario, realizando un análisis comparativo entre agentes intervinientes, en el marco de un estudio tipo encuesta de carácter descriptivo, inferencial y correlacional. Entre las buenas prácticas percibidas destaca la focalización en la persona usuaria y en sus circunstancias; al tiempo que las carencias se asocian principalmente a la transparencia de sus métodos y acciones, $y$ a la accesibilidad de los servicios. Se identifican discrepancias entre ambos grupos, con valoraciones más positivas por parte de las personas orientadoras.

PALABRAS CLAVE: ESPAÑA * EMPLEO * ORIENTACIÓN PROFESIONAL * CARRERA * ADULTO

\footnotetext{
* Departamento de Métodos de Investigación y Diagnóstico en Educación II, Facultad de Educación, Universidad Nacional de Educación a Distancia (UNED), España. mfsanchez@edu.uned.es

** Cursando Doctorado en Educación, Escuela de Doctorado de la Universidad Nacional de Educación a Distancia (UNED), España. carmengarcia26@hotmail.es

*** Departamento de Métodos de Investigación y Diagnóstico en Educación, Facultad de Ciencias de la Educación de la Universidad de Sevilla, España.

msuarez@us.es
} 


\section{ABSTRACT}

The socio-labor guidance services are a great support for the job-professional insertion of the job seekers. This research explores about the assessment that the different elements that make up the good practices of orientation in the employment services of the Region of Murcia (Spain), based on the opinions of users and counselors. A questionnaire was applied, performing a comparative analysis between intervening agents, in the framework of a survey study of a descriptive, inferential and correlational nature. Among the good practices perceived, the focus is on the user and their circumstances, while the shortcomings are mainly associated with the transparency of their methods and actions, and the accessibility of services. Also, in certain aspects discrepancies are identified, with generally higher ratings among counselors.

KEYWORDS: SPAIN * EMPLOYMENT * VOCATIONAL GUINDANCE * CAREER * ADULTS

\section{INTRODUCCIÓN}

En el panorama sociolaboral español persisten elevadas tasas de desempleo (cerca de 3,5 millones de desempleados registrados en 2018, según el Servicio Público de Empleo Estatal) a pesar de la progresiva tendencia a su reducción de los últimos años. Aunque se presenta en la actualidad un incremento de la tasa de ocupados, alcanzando los 18,9 millones de personas en 2017, según la Encuesta de Población Activa (Instituto Nacional de Estadística, 2018); no obstante, una parte de ese empleo no permite a determinados colectivos salir de la pobreza (Comité técnico de la Fundación FoEsSA, 2015; Navarro y Pastor, 2017), en la medida en que son empleos caracterizados por la inestabilidad, la precariedad y la informalidad, creando relaciones laborales que sostienen economías sumergidas y la escasa posibilidad de la persona para acceder a una formación permanente a un empleo de calidad.

Esta situación se agudiza en contextos y colectivos desfavorecidos socialmente, siendo complejo lograr un refuerzo personal/profesional de las personas demandantes de empleo con estos perfiles. En este sentido, la orientación sociolaboral $-y$ con ello la labor que realizan los servicios de orientación del contexto estudiado-, se convierte en un recurso esencial para el apoyo y el acompañamiento de estas personas en pro de desarrollar competencias que permitan mejorar su empleabilidad $y$ diseñar itinerarios de inserción laboral más óptimos, realistas y con viso de futuro.

En el escenario de una economía neoliberal globalizada, la deslocalización, la segmentación de los mercados y el cambio tecnológico acompañan la desregulación laboral y la coexistencia de una economía no formal. Este contexto alimenta la exclusión social y la pobreza de amplios colectivos - particularmente, mujeres, jóvenes, migrantes y mayores de 45 añosconstatándose cada vez más, como advierten diversos informes $y$ estudios, una tendencia al incremento de las desigualdades, mientras crece el empleo precario, se reduce el empleo decente y se recorta la protección social (European Anti Poverty Network, 2017; Organización Internacional del Trabajo, 2017; Sconfienza, 2017; Tausch, 2010; Vicent, 2017-2018).

De manera específica, la Región de Murcia es una de las 17 comunidades autónomas que configuran el Estado español. Ocupa un territorio de 11314 kilómetros cuadrados, con 45 municipios y 1470273 habitantes; de ellos, 202034 son extranjeros procedentes principalmente de América (Ecuador, Bolivia, Colombia y Perú) y de países africanos (Marruecos, Camerún y Senegal) (Instituto Nacional de Estadística, 2018). Por lo general, la población inmigrante no dispone de permiso de residencia y de trabajo, situación que provoca en la Región la existencia de trabajos de economía sumergida especialmente en tareas agrícolas y servicio 
doméstico con sueldos bajos y condiciones laborales precarias. En 2014, la economía sumergida en la Región se situó por encima del $26 \%$ del PIB regional (Sardá, 2014).

Por otro lado, la situación de crisis económica de los últimos 10 años ha conducido a esta región a tener una de las rentas medias más bajas de España y a acelerar el número de despidos laborales en las empresas, aumentando con ello la desprotección de la población en general y pasando muchos de los desempleados a formar parte de los colectivos desfavorecidos. Estas circunstancias han provocado un aumento de la tasa de pobreza.

En 2017, la Asociación Religiosa Cáritas atendió en Murcia a 73000 personas cuidadanas y de ellos, el 52,73\% son considerados pobres crónicos. A finales de 2016, la tasa de riesgo de pobreza en la Región era de 34,8 (European Anti Poverty Network, 2017) y en 2017, a pesar de la recuperación económica y la activación del empleo, un 30,1\% de sus habitantes estaban en riesgo de pobreza (Expansión-Datos macro, 2018). Los perfiles de riesgo de pobreza, tal como indican los avances de la investigación sobre riesgo de pobreza que está realizando la European Anti Poverty Network de la Región de Murcia (EAPN-RM) y la Universidad de Murcia (2018), son principalmente los jóvenes sin estudios, los trabajadores mayores de 45 años con trayectorias inestables antes de la crisis $y$ los hogares monoparentales compuestos por una madre migrante con hijos e hijas en edad escolar, situación muy común en la Región provocada por la inmigración de la última década.

Para reducir las consecuencias de la precariedad y la exclusión laboral, como parte de las denominadas "políticas activas de empleo", cada vez es más necesario que se multipliquen los esfuerzos por dotar de una mayor calidad a los servicios de orientación sociolaboral de modo que puedan ayudar con mayor eficacia en el desarrollo profesional de las personas jóvenes y adultas.

El Sistema Nacional de Empleo español comienza a desarrollarse en $1995^{1}$ y en la

1 Regulado inicialmente por el Real Decreto 735/1995 del 5 de mayo (Boletín Oficial del Estado de 8 de mayo) por el que se regulan las agencias de actualidad está integrado por el Servicio Público de Empleo Estatal (SEPE) y por una red de Servicios Públicos de Empleo (SPE) ${ }^{2}$ gestionados desde las comunidades autónomas españolas en colaboración con diversos centros (entidades sociales, asociativas y de apoyo a ciertos colectivos vulnerables, sindicales, empresariales, etc.). Estos servicios están destinados prioritariamente a las personas desempleadas jóvenes y adultas, $y$ en menor medida, a las personas ocupadas que buscan un mejor empleo. Funciona a partir de modelos estructurados de acciones que se llevan a cabo a través de entrevistas individuales $y$ acciones grupales de orientación.

Aunque estos programas vienen siendo un gran apoyo para ayudar a las personas jóvenes $y$ adultas a encontrar un empleo inmediato, por lo general, no han profundizado en un enfoque integral centrado en la construcción del proyecto profesional y vital (Chisvert, 2014; Padilla-Carmona, Sánchez-García y Suárez-Ortega, 2016), exceptuando aquellos que proporcionan los denominados Itinerarios Personalizados de Inserción (IPI), más próximos a un enfoque integral de la orientación (Climent-Rodríguez y Navarro-Abal, 2016). En este contexto, la calidad de estos servicios resulta determinante para conseguir que sus acciones tengan un impacto sobre las carreras y las vidas de las personas beneficiarias.

De acuerdo con diversos autores, la calidad de un servicio u organización viene definida por su capacidad para satisfacer las necesidades del cliente (Fontalvo y Vergara, 2010). Por tanto, este concepto está estrechamente relacionado con los usuarios y usuarias del servicio. Desde el enfoque de la "gestión de la calidad", aplicada a los servicios públicos, se remarca la finalidad de identificar y de resolver problemas, siendo criterios importantes tanto la definición de las metas del servicio, como

colocación sin fines lucrativos y los servicios integrados para el empleo.

2 Ley 56/2003 del 16 de diciembre, de Empleo (Boletín Oficial del Estado del 17 de diciembre). Modificada parcialmente por el Real Decreto-ley 3/2011 del 18 de febrero, de medidas urgentes para la mejora de la empleabilidad y la reforma de las políticas activas de empleo. 
la medición de sus acciones, del logro de sus objetivos y de su impacto en la realidad de las personas usuarias (Muñoz, 1999).

En este sentido, un asesoramiento de calidad $y$ un adecuado itinerario formativo permitirán a los usuarios y usuarias ampliar sus presentes $y$ futuras posibilidades profesionales, lograr el acceso a un puesto de trabajo y, sobre todo, aprender a gestionar su carrera vital $y$ profesional en contextos y condiciones laborales cada vez más inciertas y cambiantes. A pesar del papel estratégico y de los beneficios directos que pueden reportar este tipo de servicios (CEDEFOP, 2006), todavía son escasas las prácticas de comprobación sistemática de su calidad, tanto en relación con la suficiencia de estos como la pertinencia y la amplitud de sus acciones (Chisvert, 2014; Padilla-Carmona, Sánchez-García y Suárez-Ortega, 2016). Es preciso por ello desarrollar un esfuerzo de evaluación de la calidad de estos servicios públicos $y$, en ese marco evaluativo, identificar tanto las insuficiencias como las buenas prácticas para avanzar en su mejora, generalización y desarrollo. El presente estudio pretende contribuir en esta línea.

\section{BENEFICIOS INDIVIDUALES Y SOCIALES DE LA ORIENTACIÓN SOCIO-PROFESIONAL}

El proceso mediante el cual el individuo desarrolla su carrera profesional tiene lugar a lo largo de toda la vida personal y profesional. En el apoyo a las personas para optimizar ese desarrollo, se encuentran implicados varios agentes que aparecen en diversos momentos del desarrollo evolutivo y del contexto donde se sitúan en cada momento, entre ellos, los centros educativos y las universidades, los servicios de orientación sociolaboral, la comunidad, la empresa, etc. Ese apoyo no debería limitarse a quienes presentan fracaso académico (cuando se trata de contextos educativos), o a los demandantes de empleo (en contextos comunitarios de orientación sociolaboral), sino que debería ser accesible en cualquier momento en que una persona necesite activar su proyecto profesional, incluyendo la realización de una labor preventiva en los últimos cursos en los centros de educación secundaria, formación profesional y durante toda la etapa universitaria, formando aquellas competencias de gestión de la carrera necesarias e imprescindibles en el futuro laboral de las personas.

La orientación se puede definir como una actividad profesional de ayuda, estímulo y acompañamiento de la persona a lo largo de su vida, en aquellos contextos donde se encuentra (ya sea educativo, laboral o socio-comunitario). Su finalidad es propiciar su progresiva auto-orientación, facilitando que planifique y alcance el desarrollo satisfactorio en su trabajo y en su carrera profesional (Sánchez, 2017). El uso de los términos orientación profesional (profesional guidance) y orientación vocacional (vocational guidance) es indistinto, siendo el primero más utilizado en España y en diversos países europeos y más común, el segundo en los ámbitos latinoamericano y anglosajón. Otro término utilizado frecuentemente en España es el de orientación socio-laboral, referida a un tipo de ayuda que pone el acento en la inserción laboral y la búsqueda de empleo; objetivo central en el que se posiciona la orientación proporcionada actualmente por el Sistema Nacional de Empleo.

Uno de los objetivos centrales de la orientación es el de ayudar a la clarificación de las metas y objetivos vitales/profesionales, algo básico para la vida y el desarrollo del individuo. De ahí la importancia de contribuir, desde la acción orientadora, al desarrollo del proyecto vital y profesional de las personas, promoviendo la reflexión sobre sus inquietudes, aspiraciones $y$ oportunidades para que este proyecto sea coherente y realizable, más allá de la búsqueda puntual de un empleo (Guichard, 2013). Desde ese planteamiento, se asume cada vez más un enfoque global que favorezca la toma de decisiones, la construcción activa de itinerarios y la gestión de la carrera de forma continua, en un contexto marcado por la necesidad de adaptación a los cambios y a la incertidumbre laboral (Krumboltz, Foley y Cotter, 2013; Lent, 2013; Patton y McMahon, 2006; Savickas, 2012 y 2013; Sobrado y Ceinos, 2017). En este sentido, se resalta la importancia de detectar $y$ de impulsar las potencialidades $y$ las competencias del sujeto en el contexto en el que actúa (Álvarez 
y López, 2012; McCarthy, 2016). Sin olvidar, como indican Blanco, Ramos y Sánchez (2018), el aspecto motivacional $y$ de enriquecimiento personal que alberga la formación permanente, unida a unos itinerarios personalizados que permitan a la persona mantener su empleo $y$ en el que, como también indica el Informe de la Fundación FOESSA (2015), el personal orientador constituye una figura clave e imprescindible.

\section{NECESIDAD DE UN ENFOQUE INTEGRAL EN LA PRÁCTICA ORIENTADORA}

En el contexto específico de los servicios de orientación e inserción laboral, la adopción de un enfoque integral pasa por asegurar ciertos parámetros de calidad reconocidos por las organizaciones y especialistas del campo de la orientación educativa y profesional. En este sentido, la Asociación Internacional de Orientación Escolar y Profesional (AIOSP) $)^{3}$ desarrolló en 2006, los principios y normas éticas para asegurar una orientación educativa y profesional de calidad; planteamientos integrados por la Network for Innovation in Career Guidance y Counseling in Europe (NICE) (Schiersmann et ál., 2012) en su propuesta de puntos comunes en la formación de profesionales en Orientación.

En una línea similar, en el contexto español, se promueven estos principios y planteamientos por parte de las organizaciones profesionales del ámbito de la Orientación (COPOE, 2015). En coherencia, diversos organismos internacionales promueven iniciativas que ayudan a mejorar las prácticas en esta área. Así, el Centro Europeo para el Desarrollo de la Formación Profesional (CEDEFop) elaboró en 2006, el informe Mejorando las politicas y sistemas de orientación continua. El uso de herramientas de referencia comunes en Europa.

Las buenas prácticas pueden entenderse como el conjunto de acciones y procedimientos que resultan eficaces $y$ satisfactorios para el logro de ciertos objetivos atribuidos a una orientación de calidad. Estos son compartidos tanto

3 Para mayor información de esta asociación, visitar su página: http://iaevg.net/iaevg.org/IAEVG/ nav196f.html desde los diversos organismos internacionales (CEDEFOP, 2006, 2014a y 2014b; Consejo de Europa, 2008; OCDE, 2004 y 2006) como por parte de los investigadores y especialistas de este ámbito disciplinar (entre ellos, Alcoforado, 2013; Bartual y Turmo, 2016; Chisvert-Tarazona, 2014; Fiorini, Bardagi y Silva, 2016; Villar y Méndez, 2014; Wong, 2016). Para conseguir estas metas es preciso, por tanto, contar con las estrategias necesarias y servicios bien definidos y sustentados en una base teórica acorde a las demandas de los tiempos actuales.

Entre las buenas prácticas defendidas ocupa un lugar destacado el acceso de los ciudadanos a la orientación de forma permanente $y$ frente a diversos tipos de transiciones a lo largo de la vida. En las conclusiones de la Comisión Europea (2008), sobre nuevas capacidades para nuevos empleos, se considera prioritaria la orientación y la formación permanentes para las personas jóvenes y adultas, particularmente para aquellas que se encuentran en situación de desempleo y requieren integrarse en el mercado laboral. Por tanto, un componente de las buenas prácticas es disponer de servicios de orientación permanentes y estables, cercanos a la ciudadanía y que cuenten con recursos humanos cualificados y suficientes que garanticen la calidad del servicio (CEDEFOP, 2006).

En esa línea, las recomendaciones de la OCDE (2004) y del Consejo de Europa (2008) para garantizar la calidad de los procesos de orientación, hacen referencia a que los servicios deberían disponer de los medios y recursos suficientes, incluidos los tecnológicos, así como, bases documentales especializadas. Asimismo, deberían contar con instalaciones adecuadas y accesibles, junto con mecanismos que permitan difundir $y$ visibilizar sus acciones $y$ servicios entre la población.

Otro de los principios compartidos de forma destacada se refiere a garantizar que dicho acceso a los servicios de orientación tenga lugar en igualdad de condiciones para todas las personas, con el objetivo de reducir la desigualdad sin dejarse influenciar por condiciones como la edad, el sexo, el origen cultural, la clase social u otro motivo (AIOSP, 2013). Se 
plantea también la necesidad de un posicionamiento de la orientación profesional dentro de un contexto neoliberal, en el que se reitera el compromiso de la orientación y su importante papel para contribuir a la justicia social y a la equidad en el acceso a la educación y al empleo (Irving y Malik, 2005), de modo que pueda ejercerse una mediación entre el ciudadano y el Estado (Holey, Sultana y Thomsen, 2017; Holey y Sultana, 2016).

La orientación profesional es particularmente necesaria en los casos en los que las personas se sienten excluidas de la participación en el aprendizaje y en el mercado laboral. Por ello, la acción de los servicios de orientación para ayudarles a construir y a gestionar su proyecto profesional-vital debe adaptarse a los usuarios y a sus condicionantes, adecuando su metodología y facilitando servicios accesibles, disponibles $y$ flexibles con sus necesidades desde el máximo respeto a las diferencias individuales y entre los grupos sociales (CEDEFoP, 2014a; Consejo de Europa, 2008 y OCDE, 2004).

Resulta fundamental el papel de la Orientación para promover las competencias de gestión de la carrera, es decir, para evaluar $y$ ampliar los perfiles profesionales $y$ para propiciar la responsabilidad del individuo en su propio desarrollo profesional (CEDEFOP, 2011 y 2017). Particularmente, se ha apuntado la importancia de promover las competencias metacognitivas, de autoaprendizaje, de autoconocimiento $y$ de toma de decisiones, permitiendo que la persona participe activamente en su propio proceso de aprendizaje, permitiéndole construir diferentes tipos de competencias $y$ oportunidades en su trayectoria (Coll, Mauri y Rochera, 2012). La habilidad de aprender a aprender (Padilla-Carmona, 2013) y, estratégicamente, la de utilización de las TIC (Fernández, 2017) le permitirán enfrentar los retos del futuro, no solo en momentos de crisis, sino a lo largo de la carrera. En este sentido, el autoaprendizaje constituye una clave adaptativa para seguir ampliando y diversificando el abanico de habilidades y aprendizajes, así como seguir tomando nuevas decisiones.
Desde estos planteamientos, un reto esencial para dotar de calidad a los servicios de orientación es que ese apoyo continuo permita a la persona construir y desarrollar un proyecto profesional/vital de modo que pueda lograr una articulación coherente de sus metas con su identidad profesional y personal, a la vez que desde esa base se facilita que la persona orientada sea el agente activo de su propio proceso de autoconstrucción.

Son todavía escasos los estudios en España que analizan las prácticas de los servicios de orientación para el empleo (entre ellos: Chisvert, 2014; González y Martínez, 2016; SuárezOrtega, Padilla-Carmona y Sánchez-García, 2013; Suárez-Ortega, Sánchez-García y GarcíaGarcía, 2016). Otros trabajos analizan las necesidades de orientación de las personas usuarias (Padilla-Carmona, Sánchez-García y SuárezOrtega, 2016; Villar y Méndez, 2014), poniendo de relieve las carencias en las habilidades para gestionar la carrera y para tomar decisiones realistas en condiciones adversas.

\section{PROCEDIMIENTO METODOLÓGICO}

\section{OBJETIVOS}

Este estudio se propone indagar acerca de la valoración que merecen los distintos elementos que configuran las buenas prácticas, tanto desde el punto de vista de los usuarios que acuden a los servicios de orientación, como de los profesionales que les atienden. El objetivo es identificar las buenas prácticas, las insuficiencias y los retos pendientes en los servicios de orientación para el empleo de la Región de Murcia, y específicamente:

1) Explorar las características psicométricas del instrumento: estructura factorial y fiabilidad derivada de la aplicación en la Región de Murcia.

2) Describir las percepciones de usuarios y orientadores en relación con las buenas prácticas en los servicios de orientación profesional en este contexto.

3) Comparar las percepciones de ambos colectivos, extrayendo sus implicaciones para la mejora de la práctica orientadora. 
MÉTODO

El diseño de investigación se aborda desde un enfoque cuantitativo, realizando un estudio tipo encuesta, de carácter descriptivo, inferencial y correlacional. Este método permite obtener información comparada sobre un número de usuarios y usuarias de los servicios y un número de profesionales de dichos servicios. El estudio se enmarca en la línea investigadora abierta a través del proyecto ORIEM $^{4}$ (Orientación y Empleo), centrándose en la Región de Murcia.

\section{INSTRUMENTO}

Se aplica la Escala de valoración de criterios básicos en la prestación de servicios de orientación (EVPO), diseñada ad hoc por Padilla-Carmona, Sánchez-García y Suárez-Ortega (2011). La escala tiene dos versiones adaptadas en su redacción y dirigidas respectivamente a los usuarios y usuarias de los servicios de orientación y a profesionales de dichos servicios. En ambas versiones, los 17 ítems que las integran, corresponden con los principios (criterios) establecidos por el Centro Europeo para el Desarrollo de la Formación Profesional (CEDEFOP, 2006) para la prestación de los servicios de orientación.

El estudio que estableció dichos criterios fue desarrollado por un equipo internacional compuesto por 25 personas expertas en Orientación, procedentes de diversos países de la UE. $\mathrm{Su}$ contenido se estructura en torno a cuatro ejes: 1) la focalización en la población beneficiaria, 2) la participación activa de los ciudadanos y ciudadanas, 3) el acceso a los servicios y 4) la garantía de calidad de la orientación (CEDEFOP, 2006). Las opciones de respuesta se presentan en una escala tipo Likert de 6 puntos donde el valor 0 indica un total desacuerdo y el 5 un total acuerdo con la afirmación presentada.

4 La orientación del proyecto vital y profesional en la edad adulta: Análisis de necesidades y valoración de servicios para el empleo e identificación de buenas prácticas, subvencionado dentro del Plan Nacional de I+D+I (ref.: EDU2010-21873-C03-01).

\section{PARTICIPANTES}

Para la selección de los participantes se tuvo en cuenta la estructura organizativa de los servicios públicos de orientación profesional en la Región de Murcia, los cuales están centralizados a través del Servicio Regional de Empleo y Formación (SEF). Este se financia principalmente con fondos sociales europeos que anualmente se aprueban y se conceden al Estado español, que los distribuye entre las comunidades autónomas, entre ellas, la Región de Murcia.

El SEF dispone en la actualidad de 25 oficinas de empleo distribuidas por toda la Región y en ellas están ubicados 125 profesionales en Orientación, quienes proporcionan apoyo para desarrollar un itinerario o una ayuda personalizada a las personas desempleadas que se inscriben voluntariamente como demandantes de empleo.

Asimismo, el SEF financia - como centros colaboradores - a entidades privadas sin ánimo de lucro, sindicatos y asociaciones de personas con discapacidad para impartir orientación a colectivos de demandantes de empleo como personas desempleadas de larga duración ${ }^{5}$ o colectivos de exclusión social. En los dos últimos años, el SEF también ha subvencionado a 52 agencias privadas de colocación para insertar y orientar a las personas que acuden a sus servicios. Además, en los 45 municipios de la Región, los ayuntamientos o entidades locales también reciben a través de la Dirección General de juventud de Comunidad Autónoma, subvenciones anuales para impartir acciones de orientación laboral para jóvenes de 16 a 29 años.

En la Región de Murcia existen 4 universidades, la Universidad de Murcia (UMU), la

$5 \quad$ Personas que independientemente de la edad, perfil o profesión se encuentran desempleadas a lo largo de un periodo superior a 12 meses y están inscritas como demandantes de empleo en los servicios de empleo estatales. En el caso de personas jóvenes de hasta 29 años, se consideran desempleadas de larga duración con 6 meses de desempleo. 
Universidad Politécnica de Cartagena (UPCT), la Universidad Católica San Antonio (UCAM) y la Universidad a Distancia (UNED) con tres delegaciones. Todas disponen de un servicio de orientación profesional, exclusivamente para sus estudiantes y para quienes han egresado.
El universo de estudio está conformado por personas usuarias entre 16 y 64 años que han acudido a los servicios de empleo en la Región de Murcia para recibir orientación profesional y por las personas orientadoras que trabajan en dichos servicios (tabla 1).

TABLA 1

MUESTRA DE PERSONAS USUARIAS Y PROFESIONALES DE LOS SERVICIOS DE ORIENTACIÓN SEGÚN EDAD Y SEXO

\begin{tabular}{l|c|c|c|c|c|c}
\hline & \multicolumn{3}{|c|}{$\begin{array}{c}\text { USUARIOS Y USUARIAS } \\
\mathrm{n}=254\end{array}$} & \multicolumn{3}{c}{ ORIENTADORES Y ORIENTADORAS } \\
$\mathrm{n}=76$
\end{tabular}

Fuente: Elaboración propia.

Para la selección de las dos submuestras, primeramente se seleccionaron 6 servicios de la región, priorizando los siguientes criterios: 1) asegurar una diversidad de los servicios en cuanto a la distribución geográfica y contextual, 2) la disponibilidad para colaborar en el estudio por parte de sus responsables. Estos centros fueron: el Servicio Regional de Empleo y Formación de la Región de Murcia, la Agencia de Desarrollo Local de Alcantarilla, la Agencia de Desarrollo Local de Murcia, Escuelas Taller de la Región de Murcia y el Centro de Orientación, Información y Empleo (COIE) de la Universidad de Murcia. Una vez seleccionados los servicios, contando con la colaboración de los responsables, fue invitada la totalidad de las personas usuarias y profesionales para cumplimentar el instrumento. En el caso de profesionales, se contó además con la participación de otros pertenecientes a la Agencia de Desarrollo Local de Molina de Segura y del servicio de orientación ONCE-Fundosa ${ }^{6}$.

Se obtuvo finalmente una muestra de 254 personas usuarias y otra de 76 orientadores, pertenecientes a las localidades de Murcia, Alcantarilla, Molina del Segura, Alguazas, Cartagena, Lorquí, la Unión y Jumilla.

Respecto a la formación de quienes participaron, el $43,7 \%$ tiene estudios

$6 \quad$ La Organización Nacional de Ciegos Españoles (ONCE) nació en 1938 y es en la actualidad una corporación de carácter social sin ánimo de lucro, destinada a mejorar la calidad de vida de las personas ciegas, deficientes visuales y discapacitadas de toda España. Fundosa es un grupo empresarial dependiente de la Fundación ONCE, destinado a la integración sociolaboral de las personas con discapacidad. 
obligatorios y el 29,5\% únicamente estudios primarios, mientras que el $26,8 \%$ cuenta con estudios posobligatorios ${ }^{7}$ o universitarios. La formación más frecuente en el caso de profesionales se encuadra en el ámbito de la Psicología y la Pedagogía. La tabla 2 recoge la distribución detallada según formación y sexo.

TABLA 2

NIVEL DE ESTUDIOS DE LAS PERSONAS ORIENTADORAS ENCUESTADAS SEGÚN SEXO

\begin{tabular}{|c|c|c|c|c|c|c|}
\hline & \multicolumn{2}{|c|}{ MUJERES } & \multicolumn{2}{|c|}{ HOMBRES } & \multicolumn{2}{|c|}{ TOTAL } \\
\hline & FREC. & $\%$ & FREC. & $\%$ & FREC. & $\%$ \\
\hline Psicología & 20 & 33,9 & 6 & 35,3 & 26 & 34,2 \\
\hline Pedagogía & 19 & 32,2 & 4 & 23,5 & 23 & 30,3 \\
\hline Psicopedagogía & 1 & 1,7 & - & - & 1 & 1,3 \\
\hline Educador social & 1 & 1,7 & 1 & 5,9 & 1 & 1,3 \\
\hline Economía/ADE/Empresariales & 1 & 5,9 & 1 & 5,9 & 2 & 2,6 \\
\hline Derecho & 2 & 3,4 & - & - & 2 & 2,6 \\
\hline Sociología & 2 & 3,4 & - & - & 2 & 2,6 \\
\hline Ciencia del trabajo & 3 & 5,1 & 1 & 5,9 & 4 & 5,3 \\
\hline Magisterio & 4 & 6,8 & 2 & 11,8 & 6 & 7,9 \\
\hline Trabajo Social & 3 & 5,1 & 1 & 5,9 & 4 & 5,3 \\
\hline Bachiller/Form.Profesional & 3 & 5,1 & 2 & 11,8 & 5 & 6,9 \\
\hline TOTAL & 59 & 100 & 17 & 100 & 76 & 100 \\
\hline
\end{tabular}

Fuente: Elaboración propia.

\section{RECOLECCIÓN Y ANÁLISIS DE DATOS}

La aplicación de las escalas se realizó durante los años 2015 y 2016. En el caso de las personas usuarias se realizaron directamente en los centros $y$ entidades de orientación, $y$ en el caso de las personas orientadoras fueron enviados por vía electrónica, a través de una carta explicativa de invitación.

Para dar respuesta a los objetivos propuestos, los datos obtenidos fueron analizados utilizando el paquete estadístico sPSS v.24, realizando análisis descriptivos, factoriales (análisis factorial exploratorio), correlacionales

$7 \quad$ Posteriores a la educación obligatoria. En el sistema educativo español corresponde con estudios de Bachillerato o Formación Profesional. (alpha de Cronbach) y contrastes entre grupos (Chi cuadrado, U de Mann-Whitney). Los análisis factoriales se realizaron a través del método de Ejes principales con rotación Varimax, a fin de identificar los factores presentes $y$ el porcentaje de varianza explicado; previamente se comprobaron las medidas кмо у prueba de Bartlett, para decidir sobre la idoneidad del análisis factorial.

Previamente a la comparación de respuestas de personas usuarias y orientadoras, se aplicaron las pruebas de normalidad (Shapiro-Wilk) y de homogeneidad de varianzas (Levène), comprobándose la no normalidad de la distribución de los datos, y optando por la prueba no paramétrica U de Mann-Whitney como medida de comparación estadística a partir de las medianas. 


\section{RESULTADOS}

\section{ESTRUCTURA Y CONFIABILIDAD DEL INSTRUMENTO}

Para comprobar las características psicométricas del instrumento, primeramente se exploró la estructura factorial para ambas distribuciones (usuarios, usaurias y profesionales). Se comprobaron las medidas previas de кMO (muestra de usuarios y usuarias $=.953$; muestra de profesionales $=.819)$ y prueba de Bartlett $($ sig $=.000)$ para confirmar la idoneidad de aplicación del análisis factorial.

Los componentes rotados obtenidos a partir de las respuestas de usuarios y usuarias, $y$ profesionales se recogen en las tablas 3 y 4 . En el caso del primer grupo, se han identificado dos factores que justifican el $69,39 \%$ de la varianza acumulada total. 
TABLA 3

ESTRUCTURA FACTORIAL IDENTIFICADA A TRAVÉS DE LA EVPO USUARIOS Y USUARIAS

\begin{tabular}{|c|c|c|c|}
\hline \multirow{2}{*}{$\begin{array}{l}\text { FACTORES } \\
\quad \% \\
\text { VARIANZA }\end{array}$} & \multirow[t]{2}{*}{ NÚMERO DE ÍTEM } & \multicolumn{2}{|c|}{$\begin{array}{l}\text { COMPONENTES } \\
\text { ROTADOS* }\end{array}$} \\
\hline & & 1 & 2 \\
\hline \multirow[t]{15}{*}{$\begin{array}{c}F 1 \\
(61,62)\end{array}$} & $\begin{array}{l}\text { 1. Cuando he acudido al servicio y me han orientado, en todo momento me he } \\
\text { sentido libre de elegir y tomar mis decisiones. }\end{array}$ & .854 & .136 \\
\hline & $\begin{array}{l}\text { 2. La orientación facilitada se ajusta exclusivamente a los intereses de la persona, } \\
\text { no está influenciada por intereses del servicio de orientación, ni por intereses } \\
\text { institucionales o financieros. }\end{array}$ & .832 & .226 \\
\hline & $\begin{array}{l}\text { 4. Los ciudadanos tienen derecho a la privacidad de la información personal que } \\
\text { facilitan en el proceso de orientación. }\end{array}$ & .819 & --- \\
\hline & $\begin{array}{l}\text { 15. El servicio en el que he recibido orientación ofertaba varias actividades y } \\
\text { recursos según las necesidades de cada persona. }\end{array}$ & .813 & .351 \\
\hline & $\begin{array}{l}\text { 3. La orientación facilitada no discrimina por razón del sexo, edad, origen étnico, } \\
\text { clase social, cualificaciones, capacidad, etc. }\end{array}$ & .810 & .185 \\
\hline & 11. El personal de orientación ofrece una atmósfera acogedora a los ciudadanos. & .809 & .387 \\
\hline & 7. He podido participar activamente en el proceso de orientación. & .804 & .262 \\
\hline & $\begin{array}{l}\text { 17. El personal que facilita la orientación está bien preparado profesionalmente } \\
\text { para ocuparse de las necesidades de los usuarios. }\end{array}$ & .777 & .417 \\
\hline & $\begin{array}{l}\text { 5. La orientación facilitada promueve la igualdad de oportunidades de aprendizaje y } \\
\text { laborales de todos los ciudadanos. }\end{array}$ & .777 & .288 \\
\hline & $\begin{array}{l}\text { 12. La orientación facilitada apoya a los ciudadanos a través del conjunto de } \\
\text { transiciones de aprendizaje, laborales, sociales y personales que realizan o } \\
\text { encuentran. }\end{array}$ & .749 & .428 \\
\hline & $\begin{array}{l}\text { 13. Cualquier ciudadano tiene la posibilidad de acceder a este servicio de } \\
\text { orientación en cualquier momento de su vida. }\end{array}$ & .731 & .135 \\
\hline & $\begin{array}{l}\text { 14. La orientación facilitada está accesible de una manera flexible y fácil de utilizar, } \\
\text { por ejemplo, a través de entrevistas personales, por teléfono, por correo electrónico } \\
\text { o en el marco de actividades de promoción externa, y en momentos y lugares } \\
\text { adecuados. }\end{array}$ & .705 & .440 \\
\hline & $\begin{array}{l}\text { 16. Me han pedido mi opinión sobre el funcionamiento del servicio, con el fin de } \\
\text { mejorarlo. }\end{array}$ & .698 & .328 \\
\hline & $\begin{array}{l}\text { 6. En el proceso de orientación se considera el contexto personal, social, cultural y } \\
\text { económico de la toma de decisiones del ciudadano. }\end{array}$ & .674 & .205 \\
\hline & $\begin{array}{l}\text { 9. La orientación que me han facilitado me ha ayudado a ser capaz de planificar y } \\
\text { gestionar mi itinerario de formación y empleo. }\end{array}$ & .645 & .546 \\
\hline \multirow[t]{2}{*}{$\begin{array}{c}F 2 \\
(7,77)\end{array}$} & $\begin{array}{l}\text { 8. En el proceso de orientación, además de los ciudadanos y el servicio de } \\
\text { orientación, participan otros agentes (empresas, municipio, familia). }\end{array}$ & --- & .865 \\
\hline & $\begin{array}{l}\text { 10. Antes de empezar el proceso de orientación sabía con claridad para qué sirve } \\
\text { este servicio. }\end{array}$ & .333 & .568 \\
\hline
\end{tabular}

*Método de extracción: factorización del eje principal. Método de rotación: normalización Varimax con Kaiser.

Fuente: $\quad$ Elaboración propia. 
El primer factor (explicativo del 61,62\% de la varianza) agrupa un conjunto amplio de criterios asociados a la calidad, profesionalidad $y$ focalización en la persona orientada; mientras que el segundo con un peso más reducido en la varianza $(7,77 \%)$, se focaliza en un funcionamiento transparente $y$ conectado con el entorno social. El estudio de consistencia interna realizado a través del índice alpha de Cronbach aporta un valor global fiable $(\alpha=.951)$, así como en el primer factor $(\alpha=.958)$, pero con menos consistencia en el segundo $(\alpha=.498)$. En el caso de profesionales, se obtiene una estructura factorial más compleja, compuesta por cuatro factores que explican el $69,44 \%$ de la varianza total. 
TABLA 4

ESTRUCTURA FACTORIAL IDENTIFICADA A TRAVÉS DE LA EVPO. PERSONAS ORIENTADORAS

\begin{tabular}{|c|c|c|c|c|c|}
\hline \multirow{2}{*}{$\begin{array}{l}\text { FACTORES } \\
\quad \% \\
\text { VARIANZA }\end{array}$} & \multirow{2}{*}{ NÚMERO DE ÍTEM } & \multicolumn{4}{|c|}{$\begin{array}{l}\text { COMPONENTES } \\
\text { ROTADOS* }\end{array}$} \\
\hline & & 1 & 2 & 3 & 4 \\
\hline \multirow[t]{6}{*}{$\begin{array}{l}F 1 \\
(42,92)\end{array}$} & $\begin{array}{l}\text { 10. La naturaleza del servicio de orientación facilitado resulta } \\
\text { inmediatamente obvia para el usuario. }\end{array}$ & .858 & - & .208 & .127 \\
\hline & $\begin{array}{l}\text { 17. El personal que facilita la orientación dispone de capacidades } \\
\text { acreditadas a nivel nacional para identificar y ocuparse de las necesidades } \\
\text { de los ciudadanos y, en su caso, enviar a las personas a un servicio más } \\
\text { adecuado. }\end{array}$ & .841 & .278 & - & - \\
\hline & $\begin{array}{l}\text { 8. En el proceso de orientación, además de los ciudadanos y el servicio de } \\
\text { orientación, participan otros agentes (empresas, municipio, familia). }\end{array}$ & .741 & - & - & .228 \\
\hline & $\begin{array}{l}\text { 15. La orientación se facilita mediante una gran variedad de métodos para } \\
\text { responder a las diversas necesidades de los ciudadanos. }\end{array}$ & .639 & .468 & - & .344 \\
\hline & $\begin{array}{l}\text { 16. Los servicios de orientación se mejoran continuamente incluyendo de } \\
\text { manera regular las opiniones de los ciudadanos y se ofrecen al personal } \\
\text { posibilidades de formación permanente. }\end{array}$ & .604 & .352 & .216 & - \\
\hline & $\begin{array}{l}\text { 2. La orientación facilitada se ajusta exclusivamente a los intereses de la } \\
\text { persona, no está influenciada por intereses del servicio de orientación, ni } \\
\text { por intereses institucionales o financieros. }\end{array}$ & .597 & .355 & .249 & \\
\hline \multirow[t]{5}{*}{$\begin{array}{l}F 2 \\
(12,85)\end{array}$} & $\begin{array}{l}\text { 5. La orientación facilitada promueve la igualdad de oportunidades de } \\
\text { aprendizaje y laborales de todos los ciudadanos. }\end{array}$ & .135 & .775 & .219 & .258 \\
\hline & $\begin{array}{l}\text { 1. La orientación facilitada respeta la libertad de elección profesional y } \\
\text { desarrollo personal del usuario. }\end{array}$ & .249 & .737 & .377 & - \\
\hline & $\begin{array}{l}\text { 3. La orientación facilitada no discrimina por razón de sexo, edad, origen } \\
\text { étnico, clase social, cualificaciones, capacidad, etc. }\end{array}$ & - & .727 & .287 & - \\
\hline & $\begin{array}{l}\text { 6. En el proceso de orientación se considera el contexto personal, social, } \\
\text { cultural y económico de la toma de decisiones del ciudadano. }\end{array}$ & .367 & .591 & .198 & 497 \\
\hline & $\begin{array}{l}\text { 14. La orientación facilitada está accesible de una manera flexible y fácil de } \\
\text { utilizar, por ejemplo, a través de entrevistas personales, por teléfono, por } \\
\text { correo electrónico o en el marco de actividades de promoción externa, y en } \\
\text { momentos y lugares adecuados a las necesidades de los usuarios. }\end{array}$ & .475 & .541 & .142 & 437 \\
\hline \multirow[t]{4}{*}{$\begin{array}{l}F 3 \\
(7,53)\end{array}$} & $\begin{array}{l}\text { 4. Los ciudadanos tienen derecho a la privacidad de la información } \\
\text { personal que facilitan en el proceso de orientación. }\end{array}$ & - & .227 & .832 & .132 \\
\hline & $\begin{array}{l}\text { 11. El personal de orientación ofrece una atmósfera acogedora a los } \\
\text { ciudadanos. }\end{array}$ & .117 & .110 & .731 & .240 \\
\hline & $\begin{array}{l}\text { 7. Los ciudadanos pueden participar activamente en el proceso de } \\
\text { orientación. }\end{array}$ & .284 & .378 & .666 & .140 \\
\hline & $\begin{array}{l}\text { 9. La orientación facilitada ayuda al ciudadano a ser capaz de planificar y } \\
\text { gestionar sus itinerarios de aprendizaje y profesionales y las transiciones } \\
\text { existentes en ellos. }\end{array}$ & .239 & .337 & .633 & - \\
\hline \multirow[t]{2}{*}{$\begin{array}{l}F 4 \\
(6,14)\end{array}$} & $\begin{array}{l}\text { 13.Todos los ciudadanos tienen derecho a acceder a los servicios de } \\
\text { orientación en cualquier momento de su vida. }\end{array}$ & - & - & .209 & .795 \\
\hline & $\begin{array}{l}\text { 12. La orientación facilitada apoya a los ciudadanos a través del conjunto de } \\
\text { transiciones de aprendizaje, laborales, sociales y personales que realizan o } \\
\text { encuentran. }\end{array}$ & .467 & .152 & .267 & .612 \\
\hline
\end{tabular}

* Método de extracción: factorización del eje principal. Método de rotación: normalización Varimax con Kaiser.

Fuente: Elaboración propia. 
El primer factor $(42,92 \%$ de la varianza) agrupa 6 ítems centrados en bases de transparencia y profesionalidad al servicio de la persona, esto es, sistemas de mejora, transparencia, personal cualificado, en contacto con otros agentes sociales, con variedad metodológica, priorizando los intereses de la persona. El segundo factor $(12,85 \%$ de la varianza) reúne 5 ítems en torno a la priorización del respeto a los derechos de la persona, tales como, la privacidad, libertad, igualdad, acogida y derecho a reclamar. El tercer factor (7,53\% de la varianza) incluye otros 4 ítems que remiten a un enfoque integral y contextualizado de la práctica orientadora, promoviendo el acceso en cualquier momento vital, el apoyo en diversas transiciones y la acción contextualizada en el entorno de la persona. Finalmente, el cuarto factor $(6,14 \%$ de la varianza), incorpora únicamente 2 ítems que tienen que ver con la participación activa de la persona orientada. El valor de consistencia interna (alpha de Cronbach) obtenida en el conjunto de la escala es .919; por factores, se obtienen valores también satisfactorios, en el primero $(\alpha=.863)$, el segundo $(\alpha=.834)$ y el tercero $(\alpha=.757)$; si bien, la fiabilidad es más débil para el cuarto y último $(\alpha=.367)$.

\section{VALORACIÓN DE LAS BUENAS PRÁCTICAS POR USUARIOS, USUARIAS Y PROFESIONALES}

La puntuación media global obtenida sobre las buenas prácticas (ambas submuestras) puede considerarse alta $\left(M=4.00\right.$; ${ }_{\mathrm{DT}}=1.13$; escala de 0 a 5). En el caso de los usuarios y usuarias, puede observarse en la tabla 5 que las prácticas mejor valoradas afectan a la no discriminación de la acción orientadora (ítem 3 ), el respeto a la libertad personal en la toma de decisiones (ítem 1), la competencia del personal orientador (ítem 17), la consideración del contexto en la orientación recibida (ítem 6) y el papel de la orientación para promover la igualdad de oportunidades (ítem 5). Los ítems peor valorados afectan a los componentes del segundo factor identificado, relativos a la participación de otros agentes en el proceso de orientación, tales como, las empresas, el municipio o la familia (ítem 8); y respecto al conocimiento previo que tenían sobre lo que ofrece el servicio (ítem 10). Asimismo, valoran en menor medida, con medias inferiores a 3.5, el cumplimiento de la disponibilidad de los servicios para todos los ciudadanos (ítem 13), así como, que se tome en consideración la opinión de los usuarios y las usuarias para la mejora de los servicios (ítem 16). 
TABLA 5

VALORACIÓN DE BUENAS PRÁCTICAS POR USUARIOS, USUARIAS Y ORIENTADORES RESULTADOS DESCRIPTIVOS

\begin{tabular}{|c|c|c|c|c|c|c|c|}
\hline \multicolumn{4}{|c|}{ USUARIOS/AS $(\mathrm{N}=254)$} & \multicolumn{4}{|c|}{ ORIENTADORES/AS (N=76) } \\
\hline \multirow{2}{*}{ FACTORES } & \multirow{2}{*}{$\begin{array}{l}\text { NRO. DE } \\
\text { ÍTEM }\end{array}$} & \multicolumn{2}{|c|}{ DESCRIPTIVOS } & \multirow{2}{*}{ FACTORES } & \multirow{2}{*}{$\begin{array}{l}\text { NRO. DE } \\
\text { ÍTEM }\end{array}$} & \multicolumn{2}{|c|}{ DESCRIPTIVOS } \\
\hline & & $\mathrm{M} *$ & DT & & & $M *$ & DT \\
\hline \multirow[t]{15}{*}{$F 1$} & 1 & 4.20 & 1.38 & \multirow[t]{6}{*}{$F 1$} & 10 & 3.58 & 1.25 \\
\hline & 2 & 3.91 & 1.54 & & 17 & 3.74 & 1.22 \\
\hline & 4 & 4.04 & 1.71 & & 8 & 3.68 & 1.42 \\
\hline & 15 & 3.64 & 1.60 & & 15 & 4.07 & 1.14 \\
\hline & 3 & 4.25 & 1.45 & & 16 & 4.32 & 1.17 \\
\hline & 11 & 4.03 & 1.51 & & 2 & 4.25 & 1.15 \\
\hline & 7 & 4.00 & 1.59 & \multirow[t]{5}{*}{$F 2$} & 5 & 4.47 & .75 \\
\hline & 17 & 4.19 & 1.42 & & 1 & 4.61 & .68 \\
\hline & 5 & 4.11 & 1.41 & & 3 & 4.68 & .70 \\
\hline & 12 & 3.79 & 1.54 & & 6 & 4.46 & .84 \\
\hline & 13 & 3.41 & 1.90 & & 14 & 4.39 & .97 \\
\hline & 14 & 3.84 & 1.49 & \multirow[t]{4}{*}{ F3 } & 4 & 4.71 & .67 \\
\hline & 16 & 3.42 & 1.94 & & 11 & 4.49 & .79 \\
\hline & 6 & 4.17 & 1.07 & & 7 & 4.48 & .81 \\
\hline & 9 & 3.80 & 1.53 & & 9 & 4.21 & .97 \\
\hline \multirow[t]{2}{*}{$F 2$} & 8 & 2.40 & 1.99 & \multirow[t]{2}{*}{$F 4$} & 13 & 4.41 & 1.01 \\
\hline & 10 & 3.30 & 1.66 & & 12 & 4.20 & .96 \\
\hline
\end{tabular}

* Escala de 0 a 5 .

Fuente: Elaboración propia.

Por su parte, las personas profesionales de la Orientación valoran altamente los ítems que sitúan las prácticas centradas en la población beneficiaria, particularmente, la preservación de la libertad personal (ítem 1) y la no discriminación por razón de sexo, edad, raza, clase social, etc. (ítem $3, \mathrm{M}=4.68$ ), así como, en lo que se refiere a la garantía de privacidad en el proceso de orientación (ítem 4, M=4.71). También, las que tienen que ver con el acceso de los ciudadanos a los servicios, exceptuando el ítem 10, en cuanto a que la naturaleza del servicio de orientación resulte inmediatamente obvia para la persona usuaria $(\mathrm{M}=3.58)$. Atendiendo a los factores de contenido identificados, el primero obtiene valoraciones más dispersas, mientras que el resto de factores presenta valoraciones generalmente altas, con medias por encima de 4.20.

\section{SIMILITUDES Y DISCREPANCIAS}

En conjunto, se aprecian puntuaciones más altas por parte del personal orientador 
$(\mathrm{M}=4.36$; $\mathrm{DT}=.80)$, frente a la población usuaria $(\mathrm{M}=3.90 ; \mathrm{DT}=1.20)$ encontrándose diferencias significativas $(\mathrm{U}=6088.00, \mathrm{p}=.05)$. Por ítems, en la tabla 6 se presentan de forma comparativa los descriptivos (media y desviación típica) de ambos grupos, junto con los resultados del contraste. Se aprecian diferencias significativas en los ítems 4, 6, 8, 13 y 14, no resultando significativas las diferencias en el resto de criterios sobre las buenas prácticas.

TABLA 6

COMPARACIÓN DE MEDIAS, DESVIACIONES TÍPICAS Y PRUEBA DE CONTRASTE

\begin{tabular}{|c|c|c|c|c|c|}
\hline \multirow{3}{*}{ ITEMS } & \multicolumn{4}{|c|}{ DESCRIPTIVOS } & \multirow{3}{*}{$\begin{array}{l}\text { U DE MANN } \\
\text { WHITNEY }\end{array}$} \\
\hline & \multicolumn{2}{|c|}{ USUARIOS Y USUARIAS } & \multicolumn{2}{|c|}{ PROFESIONALES } & \\
\hline & M & DT & M & DT & \\
\hline 1 & 4.20 & 1.38 & 4.61 & .675 & NS \\
\hline 2 & 3.91 & 1.54 & 4.25 & 1.145 & NS \\
\hline 3 & 4.25 & 1.45 & 4.68 & .697 & NS \\
\hline 4 & 4.04 & 1.71 & 4.71 & .670 & $7876,000^{*}$ \\
\hline 5 & 4.11 & 1.41 & 4.47 & .750 & NS \\
\hline 6 & 4.17 & 1.07 & 4.46 & .840 & $7973,000^{*}$ \\
\hline 7 & 4.00 & 1.59 & 4.48 & .811 & NS \\
\hline 8 & 2.40 & 1.99 & 3.68 & 1.416 & $38269,000^{* *}$ \\
\hline 9 & 3,80 & 1.53 & 4.21 & .970 & NS \\
\hline 10 & 3.30 & 1.66 & 3.58 & 1.246 & NS \\
\hline 11 & 4.03 & 1.51 & 4.49 & .792 & NS \\
\hline 12 & 3.79 & 1.54 & 4.20 & .959 & NS \\
\hline 13 & 3.41 & 1.90 & 4.41 & 1.00 & $6801,000^{* * *}$ \\
\hline 14 & 3.84 & 1.485 & 4.39 & .967 & $7699,500^{* * * *}$ \\
\hline 15 & 3.64 & 1.596 & 4.07 & 1.135 & NS \\
\hline 16 & 3.42 & 1.938 & 4.32 & 1.173 & NS \\
\hline 17 & 4.19 & 1.420 & 3.74 & 1.251 & NS \\
\hline
\end{tabular}

$* \mathrm{p}=, 05 ; * * \mathrm{p}=, 001 ; * * * \mathrm{p}=, 01 ; \mathrm{NS}=\mathrm{No}$ significativa

Fuente: Elaboración propia.

Concretamente, se han encontrado discrepancias entre las valoraciones de personas usuarias y profesionales en cuanto a la garantía de privacidad de la información personal de la persona orientada (ítem 4), valorada altamente por parte de los orientadores, pero de manera más baja por parte de la persona usuaria; asimismo, respecto a la consideración de la contextualización del proceso de orientación en lo personal y lo socioeconómico y cultural (ítem 6). En vínculo con esto último, tampoco las personas usuarias perciben que en el proceso de orientación participen otros agentes sociales, tales como empresas, familia, municipio, etc. (ítem 8), frente a los profesionales que lo perciben en mayor medida. 
Otras divergencias contrastadas afectan a la accesibilidad de los servicios. Se detectan en la consideración de que estos sean accesibles a todos los ciudadanos y ciudadanas (ítem 13), $y$ en cuanto a la flexibilidad $y$ facilidad de utilización (ítem 14), donde son también los profesionales quienes más valoran su cumplimiento. Paradójicamente, y aunque las diferencias no son significativas, el ítem relativo a la competencia del personal orientador (ítem 17) ha sido mejor valorado por esta población.

\section{CONCLUSIONES Y DISCUSIÓN}

En relación con el primer objetivo, se comprueba que las dos versiones del instrumento EvPo, tienen cualidades psicométricas generalmente adecuadas en cuanto a su fiabilidad $y$ a su validez de constructo. Se obtienen estructuras factoriales diferentes para ambos grupos (usuarios, usuarias y orientadores, orientadoras), resultando más compleja en el caso del grupo profesional, algo que es coherente con su formación y conocimiento más profundo acerca de la función orientadora.

Atendiendo al segundo objetivo del estudio, cabe destacar que los aspectos más valorados como buenas prácticas presentes en la acción orientadora - tanto por la población usuaria como la orientadora - se vinculan a una focalización en la problación beneficiaria $y$ en sus circunstancias, particularmente, en lo que respecta a su libertad personal (ítem 1), a la privacidad de la información personal (ítem 4), y a la no discriminación ante circunstancias de vulnerabilidad (ítem 3). En esta línea, estudios como el de Martínez López (2009), Izquierdo y López (2013) y Climent y Navarro (2016), ponen el acento en la necesidad de que la acción orientadora se adapte a las necesidades de la persona usuaria, motivándole y ayudándole a desarrollar sus habilidades; $y$ ponen de relieve igualmente la necesidad de reducir la carga burocrática y flexibilizar al máximo el proceso de orientación.

Del análisis, se deriva la presencia de otra serie de buenas prácticas presentes, si bien de una forma más moderada, asociadas a la adopción de un enfoque integral y contextualizado de la práctica orientadora, a la participación activa de la persona usuaria en sus propios procesos de orientación (aspectos asociados a los factores 3 y 4 identificados a partir de las respuestas de orientadores $y$ orientadoras), $y$ a la profesionalidad del personal orientador; aspecto que, paradójicamente, es percibido de forma más moderada por este colectivo (ítem 17). En este sentido, al igual que en el trabajo de Suárez et ál. (2016), se subraya la importancia de asegurar la formación inicial y permanente del grupo orientador $y$ de avanzar cada vez más en una especialización que permita intervenir con calidad, particularmente, con colectivos más vulnerables.

Se desprende también del estudio que las carencias o aspectos mejorables se relacionan sobre todo con la ausencia de un funcionamiento suficientemente transparente sobre el tipo de ayuda que proporcionan los servicios de orientación profesional (ítem 10) y respecto a su conexión con el entorno social (ítem 8).

El análisis comparativo sobre las percepciones de ambos colectivos permite concluir que, en conjunto, profesionales presentan un panorama general más optimista sobre las prácticas de orientación, mientras que son los usuarios y las usuarias quienes aportan valoraciones más críticas para algunos ítems. Respecto a la necesaria privacidad de la información (ítem 4), el contraste estadístico indica que estos últimos perciben en menor medida que se esté garantizando este principio. En un sentido parecido, perciben en menor medida que para tomar decisiones se esté teniendo en cuenta el contexto en el que viven las personas orientadas (ítem 6), ni que estén participando otros agentes sociales en el proceso de orientación (ítem 8). Estudios como el de Taveira, Cardoso y Marques (2017) corroboran la importancia del soporte social para mejorar la autoeficacia y reducir el estrés decisional entre la población desempleada. Al igual que el trabajo de Izquierdo y López (2013), insistiendo en la relevante necesidad de la conexión social con el entorno y del papel mediador de estos servicios.

La visión sobre la accesibilidad de los ciudadanos y las ciudadanas a estos servicios es otro punto divergente entre los grupos (ítem 13), al igual que la flexibilidad y facilidad de 
utilización (ítem 14), que son percibidos también de manera más crítica por parte de las personas usuarias. En este sentido, cabe recordar que el marco normativo que regula la orientación para el empleo prioriza la atención de ciertos grupos en situación de vulnerabilidad, de modo que en la práctica quedan excluidos numerosos ciudadanos y ciudadanas que, aun estando empleados pueden sufrir precariedad laboral, o bien, atraviesen otro tipo de transiciones (personales, formativas, etc.) que afectan su carrera profesional. En esa misma línea, algunos trabajos corroboran esta limitación (González y Martínez, 2016; Villar y MéndezLois, 2014) y valoran la importancia de que esta accesibilidad sea de manera continua, en la diversidad de momentos críticos, a lo largo de toda su vida laboral.

Considerando las limitaciones de este estudio, futuras aproximaciones deberían ampliar el número de informantes, así como, el ámbito geográfico para ofrecer más referentes de contraste. Igualmente sería conveniente establecer con mayor precisión la validez de contenido del instrumento $y$ profundizar en el conocimiento sobre las prácticas de orientación a través de otros métodos (aproximación cualitativa) que ayuden a completar e interpretar los hallazgos.

Las líneas de mejora de los servicios de orientación para el empleo deben propiciar mayor transparencia sobre sus funciones y procesos de actuación, así como, la ampliación de la población beneficiaria, de modo que pueda generalizarse un enfoque más integral en la realización de itinerarios personalizados de formación y de empleo, facilitando así que los usuarios y usuarias gestionen su carrera y su proyecto profesional en contextos sociolaborales cambiantes e inciertos.

Una implicación de estos resultados apunta a la necesidad de avanzar en el mantenimiento de servicios más estables, que no dependan de financiamientos puntuales, dado que las necesidades de los ciudadanos y ciudadanas tampoco lo son (CEDEFOP, 2011a; Suarez et ál., 2016), en coherencia con los principios de acceso universal y flexible, que permita un apoyo en las distintas transiciones a lo largo de la vida, al tiempo que posibilita igualmente cauces estables que facilitan la colaboración y coordinación entre servicios públicos y/o privados evitando solapamientos de actuaciones. Por último, otra implicación razonable conecta con la necesidad de normalizar, en la misma medida, los procesos de evaluación sobre el impacto de las acciones, no solo en términos cuantitativos y transversales, sino también de forma longitudinal y en términos más cualitativos (Creswell, 2014) sobre el modo en que se benefician las personas.

\section{REFERENCIAS}

Asociación Internacional de Orientación Escolar y Profesional. (AIOSP). (2013). Comunicado de la Asociación Internacional de Orientación Escolar y vocacional sobre la Justicia Social, de 27 de septiembre de 2013. Montpellier, Francia. Recuperado de http://iaevg. net/iaevg.org/IAEVG/nav7879. html?lang=4\&menu=1\&submenu=9

Alcoforado, L. (2013). Estrategias, retos y recursos para los orientadores en el escenario de la educación y la formación a lo largo de la vida. En P. Figuera (Coord.), Orientación profesional transiciones en el mundo global. Innovaciones en orientación sistémica y en gestión personal de la carrera (pp. 21-50). Barcelona: Laertes.

Álvarez, P. R. y López, D. (2012). Centralidad del trabajo y estabilidad del proyecto profesional y vital. Revista Española de Orientación y Psicopedagogía, 23(1), 13-25.

Bartual M.T., Turmo, J. (2016). Educación superior y competencias para el empleo. El punto de vista de los empresarios. Revista Complutense de Educación, 27(3), 1211-1228.

Chisvert, M.J. (2014). Revisión del desarrollo de la orientación sociolaboral como política activa de empleo. Revista Española de Orientación y Psicopedagogía, 25(1), 8-24.

Climent-Rodríguez, J.A. y Navarro-Abal, Y. (2016). Nuevos retos en orientación laboral: de itinerarios personales de inserción a la construcción de marcas profesionales. Revista Española de Orientación 
y Psicopedagogía, 27(2), 126-13. Recuperado de https://dialnet.unirioja.es/ servlet/articulo? codigo $=5642670$

Coll, C., Mauri, T. y Rochera, M.J. (2012). La práctica de evaluación como contexto para ser un aprendiz competente. Profesorado. Revista de Currículum y Formación del Profesorado, 16 (1), 49-59. Recuperado de www.redalyc.org/ pdf/567/56724377004.pdf

Confederación de Organizaciones de Psicopedagogía y Orientación de España (COPOE).(2015). Código deontológico de la orientación educativa en España. $N^{o}$ 00. Colección Materiales para Orientar. Pontevedra: Confederación de Organizaciones de Psicopedagogía y Orientación en España.

Consejo de Europa. (2008). Council Resolution on better integrating lifelong guidance into lifelong learning strategies. 2905th Education. Youth and Cultur. Bruselas: Consejo de Europa de 21 de noviembre de 2008. Recuperado de http://www.consilium.europa.eu/uedocs/cms_data/docs/ pressdata/en/educ/104236.pdf

Comisión Europea (2008). Comunicación de la Comisión al Parlamento Europeo, al Consejo, al Comité Económico y Social Europeo y al Comité de las Regiones. Nuevas capacidades para nuevos empleos. Previsión de las capacidades necesarias y su adecuación a las exigencias del mercado laboral. Recuperado de http://eur-lex. europa.eu/LexUriServ/LexUriServ. do?uri=COM:2008:0868:FIN:ES:PDF

Comité técnico de la Fundación foessa (2015). Analizamos: La recuperación no ha llegado a los hogares más pobres. Fundación FOESSA, Empleo precario y protección social (pp. 3-17). Madrid: Fundación FOESSA y Cáritas Española.

Creswell, J.W. (2014). Diseño de investigación: Métodos cualitativos, cuantitativos y mixtos enfoques, $4^{\mathrm{a}}$ ed. Thousand Oaks, CA: SAGE.

European Anti Poverty Network. (2017). El estado de la pobreza. Seguimiento del indicador de riesgo de pobreza y exclusión social en España. $7^{\circ}$ Informe AROPE 2017. Resumen ejecutivo. Madrid. Recuperado de https://www.eapn.es/ estadodepobreza/ARCHIVo/documentos/Informe_AROPE_2017_Resumen_ Ejecutivo.pdf

European Anti Poverty Network de la Región de Murcia y Universidad de Murcia. (2018). Condiciones de vida y Bienestar social de la población en riesgo de pobreza y exclusión social en la Región de Murcia. Recuperado de http://www.eapnmurcia. org/wp/wp-content/uploads/2018/02/ Resumen-investigaci\%C3\%B3n-pobrezay-exclusi\%C3\%B3n-social.pdf

European Centre for the Development of Vocational Training (CEDEFoP). (2006). Mejorando las politicas y sistemas de orientación continúa. El uso de referencias comunes en Europa. Luxembourg: Office for Official Publications of the European Communities, European Centre for the Development of Vocational Training. Recuperado de www.cedefop. europa.eu/files/4045_es.pdf

European Centre for the Development of Vocational Training (CEDEFOP).(2006). Mejorando las politicas y sistemas de orientación continúa. El uso de referencias comunes en Europa. Luxembourg: Office for Official Publications of the European Communities, European Centre for the Development of Vocational Training. Recuperado de www.cedefop.europa.eu/ files/4045_es.pdf

European Centre for the Development of Vocational Training (CEDEFOP).(2011). 2012-2014. Prioridades a Medio plazo. Luxemburgo: Centro Europeo para el Desarrollo de la Formación Profesional, Oficina de Publicaciones de la Unión Europea.

European Centre for the Development of Vocational Training (CEDEFOP). (2014a). La orientación profesional en tiempos de inestabilidad: aunando los beneficios económicos, sociales e individuales. Recuperado de http://www.cedefop. 
europa.eu/en/publications www.cedefop. europa.eu/files/9094_es.pdf

European Centre for the Development of Vocational Training (CEDEFOP). (2014b). Navigating difficult waters: learning for career and labour market transitions. European Centre for the Development of Vocational Training (CEDEFop). Recuperado de www.cedefop.europa.eu/ files/5542_en.pdf

European Centre for the Development of Vocational Training (CEDEFOP). (2017). 2017-20. Programming document. Luxembourg: Pluclications Office. Recuperado de http://www.cedefop.europa.eu/en/publications-and-resources/ publications $/ 4152$

Expansión/Datos Macro (2018). Riesgo de pobreza de Murcia. Recuperado de https://datosmacro.expansion.com/demografia/riesgo-pobreza/espana-comunidades-autonomas/murcia

Fernández Rey, E. (2017). Gestión de la información y uso de las TIC en orientación profesional. En M. F. Sánchez García, Orientación para el desarrollo profesional (pp. 231-249). Madrid: UNED.

Fiorini, M. C., Bardagi, M. P., y Silva, N. (2016). Adaptabilidade de carreira: paradigmas do conceito no mundo do trabalho contemporâneo. Revista Psicologia Organizações e Trabalho, 16(3), 236-247.

Fontalvo, T.J. y Vergara, J.C. (2010). La gestión de la calidad en los Servicios ISO 9001: 2008. Recuperado de http://managementensalud.com.ar/ebooks/La_Gestion_de_ la_Calidad_en_los_Servicios_ISO90012008.pdf

González, C. y Martínez, P. (2016). Jóvenes con formación y orientación para el empleo: Un caso de la Universidad de Murcia (España) y de la Universidad Católica de Córdoba (Argentina). Revista Iberoamericana de Educación, 70(2), 145-166.

Guichard, J. (2013). Quel paradigme pour des interventions en orientation contribuant au développement d'un monde plus equitable au cours du 21ème siècle? сІом
2013, Career's guidance International Conference, France, September 24th to 27th.

Holey, T. y Sultana, R. (2016). Career guidance for social justice. Journal of the National Institute for Career Education and Counselling, 36, 2-11.

Holey, T., Sultana, R. G. y Thomsen, R. (eds.). (2017). The neoliberal challenge to career guidance-mobilising research, policy and practice around social justice. Career Guidance for Social Justice. Londres: Routledge.

Sardá, J. (2014). La economía Sumergida pasa factura. El avance del fraude en España durante la crisis. Madrid: Gestha, Fundació URV.

Instituto Nacional de Estadística. (2018). Encuesta de Población Activa (EPA). Cuarto trimestre 2017. Recuperado de http://www.ine.es/daco/daco42/daco4211/ epa0417.pdf

Instituto Nacional de Estadística. (2018). Población extranjera por nacionalidad $y$ sexo. Recuperado de http://www.ine. es/jaxi/Datos.htm?path=/t20/e245/p04/ provi $/ 10 / \&$ file $=0$ ccaa002.px

Irving, A. y Malik, B. (2005). Critical Reflections on Career Education and Guidance Promoting Social Justice within a Global Economy. London: Routledge Falmer.

Izquierdo, T. y López, O. (2013). El rol de las actitudes en la inserción laboral de los desempleados mayores de 45 años. Universitas Psychologica,12(3), 911-922

Krumboltz, J., Foley, P. y Cotter E. (2013). Applying the Happenstance Learning Theory to Involuntary Career Transitions. The Career Development Quarterly, 61, 15-25.

Lent, R.W. (2013). Career-life preparedness: Revisiting career planning and adjustment in the new workplace. The Career Development Quarterly, 61, 2-14.

Martínez, A. (2009). Los orientadores laborales. Trabajo cotidiano y efectos sobre sus públicos. Cuadernos de Relaciones Laborales, 27(2), 145-169. 
McCarthy, J. (2016). La orientación profesional y las habilidades para la gestión de la carrera. Una perspectiva internacional (49-64). En A. Manzanares y C. Sanz (Dir.), Orientación profesional. Fundamentos y estrategias (pp. 39-48). Ciudad Real: UCLM, Wolters Klumer.

Muñoz, A. (1999). La gestión de la calidad total en la administración pública. Madrid: Ediciones Díaz de Santos.

Navarro, J. y Pastor Seller, E. (2017). Desarrollo $y$ vulnerabilidad: Adolescentes en el escenario local en España. Revista Brasileira de Gestao e Desenvolvimento Regional, 13(1), 152-173.

Organización para la Cooperación y el Desarrollo Económicos (OCDE). (2004). Principios de Gobierno Corporativo de la $O C D E$. Madrid: Ministerio de Economía y Hacienda.

Organización para la Cooperación y el Desarrollo Económicos (OCDE). (2006). Orientación profesional Guía para responsables políticos. París: OECD.

Organización Internacional del Trabajo (ОIT). (2017). Informe Mundial sobre Salarios 2016-2017. La desigualdad salarial en el lugar de trabajo. Ginebra: Organización Internacional del Trabajo.

Padilla-Carmona, M.T., Sánchez-García, M.F. y Suárez-Ortega, M. (2011). Cuestionario de necesidades de orientación profesional de personas adultas (NOPPA). La orientación del proyecto vital y profesional en la edad adulta: análisis de necesidades, valoración de servicios para el empleo e identificación de buenas prácticas (Proyecto I+D+I 2010-2013, Ministerio de Ciencia e innovación. Referencia EDU2010-21873-C03-01). Documento inédito.

Padilla-Carmona, M.T. (2013). Aprender a aprender a lo largo de la vida. En Sánchez García, M.F. (Coord.), Orientación Profesional y Personal (pp.166-175). Madrid: unED.

Padilla-Carmona, M.T., Sánchez-García, M.F. y Suárez-Ortega, M. (2016). Necesidades de orientación profesional de usuarios de servicios públicos de empleo en España. Revista Brasileira de Orientação Proffissional, 17(2),151-162.

Patton, W. y McMahon, M. (2006). Career Development and System Theory (Second Edition). Rotterdam: Sense Pub.

Sánchez García, M. F. (2017). Marco conceptual de la orientación para el desarrollo profesional y personal. En M.F. Sánchez García (Coord.), Orientación profesional y personal (pp. 19-44). Madrid: Universidad Nacional de Educación a Distancia.

Savickas, M. (2012). Life Design: A Paradigm for Career Intervention in the 21st Century. Journal of Counseling and Development, 90(1), 13-19.

Savickas, M. L. (2013). The theory and practice of career construction. In S. D. Brown \& R. W. Lent (Eds.), Career development and counselling: Puttingtheory and research to work ( $2^{\text {a }} \mathrm{Ed}$.) (p. 147-183). Hoboken: Wiley.

Schiersmann, C., Estelt, B.J., Katsarov, J., Mulvey, R., Reid, H., \& Weber, P. (2012). Manual NICE para la Formación Académica de Profesionales de la Orientación y Asesoramiento de la Carrera. Puntos comunes de referencia. Heidelberg, Germany: Red para la Innovación en la Orientación y el Asesoramiento de la Carrera en Europa, Heidelberg University.

Sconfienza, M.E. (2017). Pobreza y acceso al empleo en Argentina ¿Cómo se relacionan demanda e inclusión laboral? Revista de Ciencias Sociales, 151, 99-123.

Servicio Público de Empleo Estatal (SEPE). (2018). Evolución del paro registrado según sectores. Últimos 10 años. Madrid: Servicio Público de Empleo Estatal. Recuperado de https://www.sepe.es/contenidos/que_es_el_sepe/estadisticas/datos_ avance/pdf/empleo/evolparoseries.pdf

Sobrado, L.M. y Ceinos, C. (2017). Modelos emergentes en orientación profesional (pp. 67-102). En M.F. Sánchez-García (Coord.), Orientación para el Desarrollo Profesional. Madrid: UNED. 
Suárez-Ortega, M., Padilla-Carmona, M.T. y Sánchez-García, M.F. (2013). Factores condicionantes del desarrollo de buenas prácticas en servicios de orientación de personas adultas. Revista Iberoamericana para la Investigación y el Desarrollo Educativo, 11. Recuperado de ride.org.mx/1-11/index. php/RIDESECUNDARIO/article/download/630/617

Suárez, M., Sánchez, M.F. y Garcia, M.C. (2016). Caracterización de buenas prácticas $y$ necesidades de mejora en los servicios de orientación para el empleo. Revista Española de Orientación y Psicopedagogía, 27(3),43-60.

Tausch, A. (2010). Globalización y desempleo. Reflexiones empíricas desde la perspectiva de la teoría del "sistema mundial". Revista de Ciencias Sociales, 128-129, 71-86.
Taveira, M.C., Cardoso, B. y Marques, C. (2017). Fatores sociocognitivos e resultados da gestão pessoal da carreira em desempregados portugueses. Revista de Estudios e Investigación en Psicología y Educación, 07, 113-117.

Vicent, L. (2017/18). Tiempos de precariedad. Una mirada multidimensional a la cuestión precaria. Papeles de relaciones ecosociales y cambio global, 140, 35-49.

Villar, M. y Méndez-Lois, M.J. (2014). Necesidades de orientación profesional de las mujeres gallegas en función de la edad. Revista Española de Orientación y Psicopedagogía, 25(2) 127-138.

Wong, S.C. (2016). Transformation of employment patterns and need for career services in Modern Singapur. The Career Development Quarterly, 64, 287-299.

Fecha de ingreso: 18/05/2018 Fecha de aprobación: 26/11/2018 